\title{
PREVALENCE AND ASSOCIATED RISKS FOR METABOLIC SYNDROME IN NIGERIANS WITH TYPE 2 DIABETES MELLITUS
}

\author{
Soyoye DO ${ }^{1}$, Adebayo $\mathrm{OJ}^{2}$, Kolawole BA ${ }^{2,3}$, Ikem RT ${ }^{2,3}$ \\ 1 Department of Medicine, Ladoke Akintola University of Technology Teaching \\ Hospital, 2 Department of Medicine, Obafemi Awolowo University Teaching Hospital, \\ 3 Department of Medicine, Obafemi Awolowo University
}

INTRODUCTION - Diabetes mellitus is considered a cardiovascular risk equivalent. The presence of other cardiovascular risk factors in patients with Diabetes may imply synergy for the occurrence and severity of cardiovascular morbidities and mortalities. Metabolic syndrome is the presence of these clusters of cardiovascular risks in an individual. Metabolic syndrome has been shown to be associated with some traditional risk factors and emerging risk factors.

AIM - This study determined the prevalence of Metabolic Syndrome defined by the International Diabetes Federation (IDF) criteria $^{1}$ in people with Type 2 Diabetes Mellitus, and also determined its associations.

METHOD - A case-control study involving 150 Nigerians with Diabetes and 150 age and sex matched controls. Relevant clinical and anthropometric measurements were taken and laboratory investigations were done. Statistical analysis was done using SPSS 14. Regression analysis was done to determine the factors associated with Metabolic Syndrome and regression results were interpreted using odds ratio and confidence intervals, $\mathrm{p}$ value of $<0.05$ was considered statistically significant.

RESULTS - The mean \pm SD age of the diabetic and control groups were $56.12( \pm 7.65)$ years and 55.76 $( \pm 7.49)$ years respectively $(\mathrm{p}=0.681)$. Prevalence of Metabolic syndrome among diabetics and control were $68.7 \%$ and $14.7 \%$ respectively. Commonest abnormality among the study subjects was reduced high density lipoprotein levels. Table 1 shows distribution of subjects with abnormal parameters. 
Table 1: Distribution of study subjects with abnormal parameters defining Metabolic Syndrome.

\begin{tabular}{|c|c|c|c|c|c|c|c|}
\hline \multirow{3}{*}{ PARAMETER } & \multicolumn{3}{|c|}{ Diabetics } & \multicolumn{3}{|c|}{ Controls } & \multirow{2}{*}{$\begin{array}{l}\text { p value } \\
\text { (Diabetics } \\
\text { vs } \\
\text { Controls) } \\
\end{array}$} \\
\hline & $\begin{array}{c}\text { All } \\
\mathrm{n}=150\end{array}$ & $\begin{array}{l}\text { Male } \\
\mathrm{n}=66\end{array}$ & $\begin{array}{l}\text { Female } \\
\mathrm{n}=84\end{array}$ & $\begin{array}{c}\text { All } \\
\mathrm{n}=150\end{array}$ & $\begin{array}{l}\text { Male } \\
\mathrm{n}=68\end{array}$ & $\begin{array}{l}\text { Female } \\
\mathrm{n}=82\end{array}$ & \\
\hline & \multicolumn{3}{|c|}{ Number $(\%)$} & \multicolumn{3}{|c|}{ Number $(\%)$} & \\
\hline Reduced HDL & $117(78.0 \%)$ & $40(26.7 \%)$ & $77(51.3 \%)$ & $101(67.4 \%)$ & $37(24.7 \%)$ & $64(42.7 \%)$ & 0.052 \\
\hline Elevated TG & $8(5.3 \%)$ & $3(2.0 \%)$ & $5(3.3 \%)$ & $2(1.3 \%)$ & $0(0 \%)$ & $2(1.3 \%)$ & 0.103 \\
\hline Elevated FPG & $116(77.4 \%)$ & $49(32.7 \%)$ & $67(44.7 \%)$ & $8(5.3 \%)$ & $5(3.3 \%)$ & $3(2.0 \%)$ & $<0.001$ \\
\hline Elevated BP & $104(69.3 \%)$ & $44(29.3 \%)$ & $60(40.0 \%)$ & $33(22.0 \%)$ & $15(10.0 \%)$ & $18(12.0 \%)$ & $<0.001$ \\
\hline Elevated WC & $112(74.7 \%)$ & $33(22.0 \%)$ & $79(52.7 \%)$ & $88(58.7 \%)$ & $19(12.7 \%)$ & $69(46.0 \%)$ & 0.005 \\
\hline
\end{tabular}

HDL = High density lipoprotein, $\mathbf{T G}=$ Triglycerides, $\mathbf{F P G}=$ Fasting plasma glucose, $\mathbf{B P}=\mathbf{B l o o d}$ pressure, WC $=$ Waist Circumference

There was no statistically significant difference in glycated haemoglobin levels among diabetic patients with Metabolic Syndrome compared with those without Metabolic Syndrome $(\mathrm{p}=0.418)$. Table 2 shows univariate analysis of risk variables for metabolic syndrome

Table 2 - Univariate analysis of risk factors and Metabolic Syndrome

\begin{tabular}{|l|l|l|l|}
\hline \multicolumn{1}{|c|}{ Parameter } & $\begin{array}{l}\text { Subjects with } \\
\text { Metabolic Syndrome } \\
(\mathrm{n}=125) \\
\text { Mean } \pm \text { SD }\end{array}$ & $\begin{array}{l}\text { Subjects without } \\
\text { Metabolic Syndrome } \\
(\mathrm{n}=175) \\
\text { Mean } \pm \text { SD }\end{array}$ & p value \\
\hline Age $($ years $)$ & $56.32 \pm 7.56$ & $55.67 \pm 7.57$ & 0.463 \\
\hline BMI $\left(\mathrm{kg} / \mathrm{m}^{2}\right)$ & $29.23 \pm 4.61$ & $25.03 \pm 4.13$ & $<0.001$ \\
\hline WC $(\mathrm{cm})$ & $98.96 \pm 9.51$ & $87.55 \pm 9.84$ & $<0.001$ \\
\hline SBP $(\mathrm{mmHg})$ & $133.24 \pm 16.63$ & $119.48 \pm 13.89$ & $<0.001$ \\
\hline DBP $(\mathrm{mmHg})$ & $82.53 \pm 10.96$ & $75.32 \pm 9.14$ & $<0.001$ \\
\hline FPG $(\mathrm{mmol} / \mathrm{L})$ & $7.11 \pm 3.01$ & $5.21 \pm 2.71$ & $<0.001$ \\
\hline TC $(\mathrm{mmol} / \mathrm{L})$ & $4.23 \pm 0.99$ & $3.97 \pm 0.81$ & 0.014 \\
\hline HDL $(\mathrm{mmol} / \mathrm{L})$ & $0.98 \pm 0.34$ & $0.86 \pm 0.32$ & 0.102 \\
\hline $\begin{array}{l}\text { Twbc } \\
\text { cells } / \mathrm{mm}{ }^{3}\end{array}$ & $5.72 \pm 1.27$ & $5.23 \pm 1.10$ & $<0.001$ \\
\hline hs-CRP $(\mathrm{mg} / \mathrm{L})$ & $1.32 \pm 1.56$ & & \\
\hline
\end{tabular}

BMI = Body mass index, $\mathrm{WC}=$ Waist circumference, $\mathrm{SBP}=$ Systolic blood pressure, DBP = Diastolic blood pressure, FPG = Fasting plasma glucose, $\mathrm{TC}=$ Total cholesterol, $\mathrm{HDL}=$ High density lipoprotein, Twbc $=$ Total white blood cell, hs-CRP $=$ high sensitivity $\mathrm{C}$-reactive protein 
Multivariate analysis showed that female gender (OR 11.556, 95\% CI 1.708 78.175), hypertension (OR 0.022, 95\% CI 0.005 - 0.106), and abdominal obesity (OR $0.861,95 \%$ CI $0.761-0.974$ ) were associated with increased odds for metabolic syndrome.

DISCUSSION - Serum levels of traditional risk factors (except high-density lipoprotein) and emerging risk factors for metabolic syndrome were found to be significantly higher in people with Metabolic Syndrome. Female gender, hypertension and abdominal obesity were associated with Metabolic Syndrome.

CONCLUSION - Metabolic Syndrome is common among our Diabetic patients. Detection and management of its cluster of risks may be an essential component in reducing its occurrence.

\section{REFERENCES}

1. Alberti KG, Zimmet P, Shaw J. Metabolic syndrome-a new world-wide definition. A Consensus Statement from the International Diabetes Federation. Diabetes Medicine 2006 May;23(5):469-80. 